\title{
Improved Visualization of the Anterior Thalamic Radiation in Bipolar Disorder by Diffusion Tensor Tractography
}

\author{
Richi Niida ${ }^{1}$, Akihiko Uechi' ${ }^{2}$, Akira Niida ${ }^{3}$, Hiroshi Matsuda ${ }^{4}$, Bun Yamagata ${ }^{1}$, Masaru Mimura $^{1}$ \\ ${ }^{1}$ Department of Neuropsychiatry, School of Medicine, Keio University, Shinjuku, Japan \\ ${ }^{2}$ Cognitive Neuroscience Research Project, Kansai Gaidai University, Hirakata, Japan \\ ${ }^{3}$ Department of Radiology, Nanbu Hospital, Itoman, Japan \\ ${ }^{4}$ Integrative Brain Imaging Center, National Center of Neurology and Psychiatry, Kodaira, Japan \\ Email: *ntrichi@yahoo.co.jp
}

How to cite this paper: Niida, R., Uechi, A., Niida, A., Matsuda, H., Yamagata, B. and Mimura, M. (2018) Improved Visualization of the Anterior Thalamic Radiation in Bipolar Disorder by Diffusion Tensor Tractography. Case Reports in Clinical Medicine, 7, 505-512.

https://doi.org/10.4236/crcm.2018.79044

Received: August 18, 2018

Accepted: September 11, 2018

Published: September 14, 2018

Copyright $\odot 2018$ by authors and Scientific Research Publishing Inc. This work is licensed under the Creative Commons Attribution International License (CC BY 4.0).

http://creativecommons.org/licenses/by/4.0/

c) (i) Open Access

\begin{abstract}
Diffusion tensor imaging (DTI) is a method for noninvasively measuring and imaging the diffusion of water molecules using MRI. Diffusion tensor tractography (DTT) can be used to presume macroscopic nerve fascicle distribution by determining, based on information obtained by DTI, whether the diffusion anisotropy vectors of adjacent voxels are continuous or not. We prepared a DTT map of the cerebral white matter nerve fascicles and investigated its usefulness for differentiation between Alzheimer's disease and bipolar disorder (BD), a major depressive disorder, based on the nerve fiber depiction, in addition to the fractional anisotropy and apparent diffusion coefficient values. We encountered two cases of $\mathrm{BD}$ in which the DTT image of the anterior thalamic radiation (ATR) improved following effective treatment with lamotrigine. Lamotrigine increases the synaptic strength due to its effect of promoting the traffic of the $\alpha$-3-hydroxy-5-methyl-4-isoxazole propionic acid (AMPA) receptor on the neuronal membrane. Downregulation of postsynaptic AMPA receptor expression can reportedly be observed during depressive episodes in BD. We consider that visualization of improvement of the ATR in response to treatment with lamotrigine (related to its pharmacological effect on the AMPA receptor) is valuable, as it suggests reversibility of the damage of the nerve fibers in this disease condition.
\end{abstract}

\section{Keywords}

Diffusion Tensor Tractography, Bipolar Disorder, Diffusion Tensor Imaging, Magnetic Resonance Imaging, Anterior Thalamic Radiation 


\section{Introduction}

$\mathrm{BD}$ is characterized by a long depressive phase, and particularly in cases with bipolar type 2 disorder in remission, the depressive state exceeds 50\% and treatment of the extended depressive state remains an issue [1] [2]. With advances in the field of diagnostic imaging, numerous studies have utilized diffusion tensor imaging (DTI) to discuss the white matter integrity in $\mathrm{BD}$, as well shown reduced FA values of the nerve fiber bundles in the anterior thalamic radiation (ATR), based on analysis of DTI [3] [4].

The ATR is a nerve fiber bundle connecting the dorsomedial nucleus of the thalamus to the prefrontal cortex; it is an important part of the so-called executive function circuit (cortico-striato-pallido-thalamic circuit) which connects the thalamus to the prefrontal cortex [5].

From our clinical experience, poor ATR visualization is an oft-recognized characteristic of BD on DTT.

In a previous paper, we reported that while the ATR can be visualized well in cases of MDD, it is poorly visualized in cases of $\mathrm{AD}$ [6]. In this report, we provide an account of the improvement of the subjective symptoms along with that of MRI visualization of the ATR following appropriate medical treatment in patients with $\mathrm{BD}$ with poor MRI visualization of the ATR prior to treatment.

\section{Data Acquisition}

The anterior thalamic radiation (ATR), cingulate fasciculus (CF), uncinate fasciculus (UF), superior longitudinal fasciculus (SLF), inferior longitudinal fasciculus (ILF), inferior fronto-occipital fasciculus (IFOF), and Fornix (FX) were selected as the nerve fascicles to be traced. We performed DTT in about 2300 patients during the 6-year period from 2012 to 2018. In this study, the DTI data were transferred to the Philips Extended MR Work Space (Release 2.6.3.2) and analyzed using the Philips Fiber Trak (Release 2.6.3.2; Koninklijke Philips Electronics NV, Amsterdam, The Netherlands), a software useful for fiber tracking. The default settings were as follows: step width for nerve fiber tracking: $10 \mathrm{~mm}$; conditions for termination of the fiber tracking: an FA value of $<0.15$ and a flip angle of $>27$ degrees. Using the multiple region-of-interest (ROI) approach and based on a tractographic atlas, Include-ROIs (to select fibers perforating the set region) were set up manually [7]. Exclude-ROIs (to exclude fibers perforating the set region) were also set for precluding other immixed nerve fibers.

Although there is involvement of the corticospinal tract and corticopontine tract in the ATR, it is feasible to achieve pure visualization of the ATR excluding fibers of the corticospinal tract and corticopontine tract by setting up an Exclude-ROI encompassing the nerve tracts of the midbrain (Figure 1). We defined the FA value as the average FA value for the entire ATR. The white matter structure peripheral to the ATR was visually assessed. In addition, visual evaluation of the ATR was defined as good when the nerve fiber fascicles of the ATR reached the boundary between the gray matter and white matter, and as poor 
when not even a single nerve fascicle of the ATR reached that boundary line.

\section{Case Presentations}

\subsection{Case 1: A 57-Year-Old Female Patient}

The patient developed symptoms of depression first when she was in her 40's. She was hospitalized for treatment with symptoms of major depression and suicidal ideation at the age 48 years. She was hospitalized a total of three times. In year X she presented with mania and a return of the depressive state. She began to be treated for $\mathrm{BD}$, but stopped taking her prescribed medication, which led to repeated manic episodes. In $\mathrm{X}+4$ years, during a major depressive episode, her oral prescription was changed from lithium to lamotrigine, as the patient complained that the lithium was causing edema. In $\mathrm{X}+5$ years, a repeat MRI showed improved visualization of the ATR. She continued to remain in a stable state, with no manic symptoms. In $\mathrm{X}+6$ years, her mental condition was still stable under continued monotherapy with $200 \mathrm{mg}$ of lamotrigine.

\subsubsection{The Results of Psychological Assessments}

The Patient Health Questionnaire-9 (PHQ9) [8] score was 27 points (full score) at the initial examination, while at the assessment conducted 5 years later, it had improved to 5 points. Assessment of cognition by the Mini-Mental State Examination (MMSE) revealed a normal cognitive state, with a score of 30 points.

\subsubsection{The Results of MRI Examinations}

Visualization of the ATR was poor on the left side in the X year, while it was good in $\mathrm{X}+6$ years (Table 1) (Figure 2 ).

\subsection{Case 2: A 63-Year-Old Male Patient}

The patient had undergone a coronary stent placement after sustaining myocardial infarction at the age of 52 years. He was identified as having BD in the same year during a hospital visit, and was advised to seek psychiatric consultation, although he did not follow the advice.

Table 1. Changes of the FA and ADC values of the ATR in the two cases examined.

\begin{tabular}{|c|c|c|c|c|c|}
\hline \multirow{2}{*}{ Case } & \multirow{2}{*}{ Examination date } & \multicolumn{2}{|c|}{ FA value } & \multicolumn{2}{|c|}{ ADC value $\left(\times 10^{-3} \mathrm{~mm}^{2} / \mathrm{s}\right)$} \\
\hline & & Right & Left & Right & Left \\
\hline \multirow{3}{*}{1} & $\mathrm{X}$ years & 0.421 & 0.423 & 0.831 & 0.822 \\
\hline & $X+5$ years & 0.440 & 0.442 & 0.826 & 0.867 \\
\hline & $X+6$ years & 0.453 & 0.434 & 0.824 & 0.819 \\
\hline \multirow{2}{*}{2} & $\mathrm{X}$ years & 0.460 & 0.481 & 0.872 & 0.829 \\
\hline & $X+9$ months & 0.434 & 0.439 & 0.918 & 0.894 \\
\hline
\end{tabular}

Abbreviations: ADC, apparent diffusion coefficient; ATR, anterior thalamic radiation; FA, fractional anisotropy. 

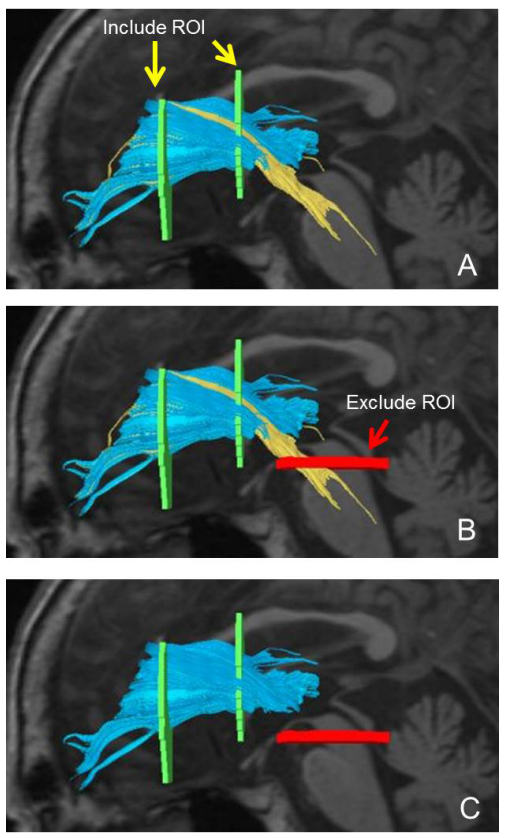

Figure 1. Sagittal T1-weighted image of the 58-year-old female patient with bipolar disorder overlaid with a diffusion tensor tractographic image of the left anterior thalamic radiation (ATR). A: The Include-region of interest (ROI) (yellow) is set on the coronal plane passing through the anterior commissure and the center of the genu of the corpus callosum. The ATR (light blue) passing through both is depicted together with the corticospinal tract and the cortical bridge (pink color). B: By setting the Exclude-ROI (red) on the cross-sectional plane passing the midbrain, the corticospinal tract and cortical bridges (pink color) traversing the same region were excluded. C: The ATR (light blue) is clearly visualized.

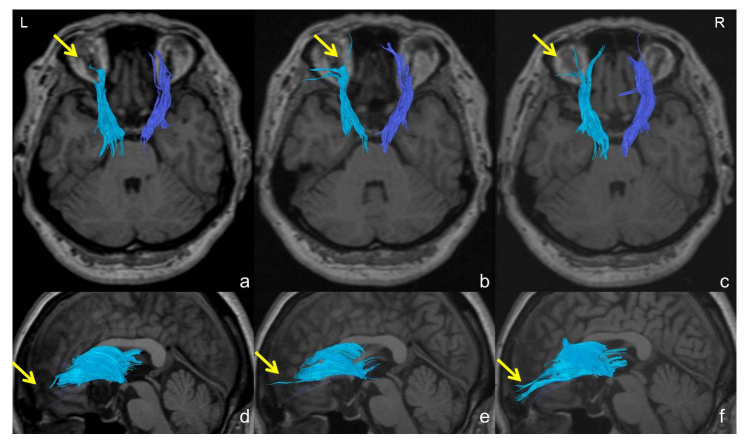

Figure 2. Diffusion tensor tractography of the anterior thalamus radiation (ATR) of Case 1 (right: blue, left: light blue). a, d: Year X (Initial consultation), b, e: X +5 years, c. f: X + 6 years. Improved visualization of the ATR in $X+5$ years $(b, e)$ as compared to that in year X (a, d). Good visualization maintained in $X+6$ years ( $c, f)$ (yellow arrows).

At the age of 62 , he was in a state of major depression. He was started on treatment during the depressed phase of BD. In January, $\mathrm{X}$ year, he was given a prescription of lamotrigine $25 \mathrm{mg}$. When the dose of lamotrigine needed exceeded $100 \mathrm{mg}$, it was increased directly to $200 \mathrm{mg}$ daily, and then $300 \mathrm{mg}$ daily. Half a year after the treatment was started, the dose was at $300 \mathrm{mg}$. By X +9 months later, the patient had recovered from a bedridden state to one where he 
could perform his daily activities and shop for himself.

\subsubsection{The Results of Psychological Assessments}

The PHQ9 was 27 points (full score) at the initial examination, while at the assessment conducted after 2 months, it had improved to 23 points, and at the assessment conducted after 1 year, it had improved further to 7 points. Assessment of cognition by the MMSE revealed normal cognitive function, with a score of 30 points.

\subsubsection{The Results of MRI Examinations}

Visual assessment revealed improvements in both the left and right ATRs (Table 1) (Figure 3).

\section{Discussions}

\subsection{Visual Confirmation of the Reversibility of ATR in Cases of BD}

There is a research which reports that the average fractional anisotropy (FA) value of the ATR has decreased in BD [9].

However, as shown in the previous section, there was no consistency in the FA value and the apparent diffusion coefficient (ADC) value of the ATR (Table 1) in the two BD cases. The FA and ADC values assessed by DTI represent the average state of the nerve fiber bundles that can be tracked. Untracked areas are not included in the evaluation, with the result being that a greater portion of the injured areas of the fiber bundles is not assessed. However, the visual evaluation method based on DTT can be used to visualize the fibers radiating from the thalamus to the surface of the brain-in short, up to the point at which the density of the nerve fiber voxels becomes very low. Consequently, it was possible to capture minute changes in the nerve fibers, and the nerve fibers that could not be captured were considered to be injured. We were able to visually confirm reversibility of the ATR visualization following treatment.

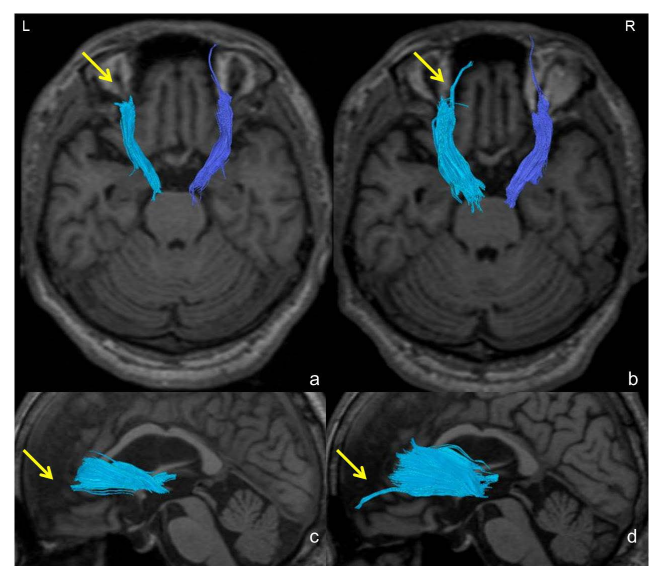

Figure 3. Diffusion tensor tractography of the anterior thalamus radiation (ATR) of Case 2 (right: blue, left: light blue). a: Year X (Initial consultation), b: 9 months later. Improved visualization of the ATR in $\mathrm{X}+9$ months (b) as compared to that in year $\mathrm{X}$ (a) (yellow arrows). 


\subsection{Pharmacological Effect of Lamotrigine (Association with the AMPA Receptor) and Improvement in Visualization of the Anterior Thalamic Radiation}

In the two BD cases, where the anterior thalamic radiation (ATR) of DTT was improved, lamotrigine was effective. It has been suggested that neurological dysfunction of the glutamate system is involved in the pathophysiology of BD [10]. Ion channel-type glutamate receptors involved in excitatory neurotransmission are classified into three types: the N-methyl-d-aspartate (NMDA), AMPA, and kainic acid receptors [11]. Lamotrigine suppresses the $\mathrm{Na}$ channel in a voltage-dependent and frequency-dependent manner, stabilizing the neuronal membranes, with consequent suppression of glutamate release. It exhibits neuroprotective activity through suppression of glutamate release and induction of apoptosis in the medial prefrontal cortex; it has been shown to ameliorate pre-pulse inhibition deficit in an animal model of mania/schizophrenia [12]. Deficit of prepulse inhibition involves dysfunction of the control of sensory-motor information and is considered to be related to impairment of cognitive function due to abnormalities in attention [13]. In addition, the functional performance circuitry, the cortico-striato-pallido-thalamic circuit, is thought to be related to pre-pulse inhibition [14]. Together, these findings suggest improved function of the cortico-striato-pallido-thalamic circuit as a possible reason for the improved visualization of the ATR following treatment with lamotrigine. It has been reported that downregulation of postsynaptic AMPA receptor expression is observed during depressive episodes of $\mathrm{BD}$; in contrast to lithium and valproic acid, lamotrigine increases synaptic strength due to its traffic-promoting activity of the AMPA receptor on the neuronal membrane, and may thereby improve depressive episodes [15]. Synaptic plasticity is related to learning and memory with long-term potentiation and increased efficiency of synaptic conduction due to increased firing of the pre-synaptic and post-synaptic cells which are thought to be involved. Increased expression of postsynaptic AMPA receptors is thought to be important for long-term potentiation [16].

The current observation of neural plasticity by DTT is considered to be a valuable demonstration of the neuroprotective effect of lamotrigine and of the importance of AMPA receptors. However, it is also important to note reports of the side effects of lamotrigine, including rash, dizziness, diplopia, and lymphadenopathy [17].

\section{Conclusion}

In this case series, using MRI based DTT, we have reported poor visualization of the ATR in BD which was not only improved in mental symptoms following appropriate medical treatment but also showed good visualization of the ATR in follow-up MRI examinations. This suggests that poor visualization of the ATR can be identified as an indication of impaired integrity of neuronal myelin of the brain in BD. ATR visualization by DTT, which makes nerve fiber plasticity pa- 
thology visible, may also be a useful and convenient method that can be implemented quickly in the clinical setting in the near future.

\section{Informed Consent}

Informed consent for participation in the study was obtained from both patients.

\section{Acknowledgements}

We are grateful to Takako Kuniyoshi of Keio University for her encouragement and administrative support.

\section{Conflicts of Interest}

The authors declare no conflicts of interest regarding the publication of this paper.

\section{References}

[1] Judd, L.L., Akiskal, H.S., Schettler, P.J., Endicott, J., Maser, J., Solomon, D.A., Leon, A.C., Rice, J.A. and Keller, B.B. (2002) The Long-Term Natural History of the Weekly Symptomatic Status of Bipolar I Disorder. Archives of General Psychiatry, 59, 530-537. https://doi.org/10.1001/archpsyc.59.6.530

[2] Judd, L.L., Akiskal, H.S., Schettler, P.J., Coryell, W., Endicott, J., Maser, J.D., Solomon, D.A., Leon, A.C. and Keller, M.B. (2003) A Prospective Investigation of the Natural History of the Long-Term Weekly Symptomatic Status of Bipolar II Disorder. Archives of General Psychiatry, 60, 261-269.

https://doi.org/10.1001/archpsyc.60.3.261

[3] Karababa, I.F., Bayazıt, H., Kılıçaslan, N., Celik, M., Cece, H., Karakas, E. and Selek, S. (2015) Microstructural Changes of Anterior Corona Radiata in Bipolar Depression. Psychiatry Investigation, 12, 367-371. https://doi.org/10.4306/pi.2015.12.3.367

[4] Chan, W.Y., Yang, G.L., Chia, M.Y., Woon, P.S., Lee, J., Keefe, R., Sitoh, Y.Y., Nowinski, W.L. and Sim, K. (2010) Cortical and Subcortical White Matter Abnormalities in Adults with Remitted First-Episode Mania Revealed by Tract-Based Spatial Statistics. Bipolar Disorder, 12, 383-389. https://doi.org/10.1111/j.1399-5618.2010.00829.x

[5] Sui, J., Pearlson, G.D., Du, Y., Yu, Q., Jones, T.R., Chen, J., Jiang, T., Bustillo, J. and Calhoun, V.D. (2015) In Search of Multimodal Neuroimaging Biomarkers of Cognitive Deficits in Schizophrenia. Biological Psychiatry, 78, 794-804. https://doi.org/10.1016/j.biopsych.2015.02.017

[6] Niida, A., Niida, R., Kuniyoshi, K., Motomura, M. and Uechi, A. (2013) Usefulness of Visual Evaluation of the Anterior Thalamic Radiation by Diffusion Tensor Tractography for Differentiating between Alzheimer's Disease and Elderly Major Depressive Disorder Patients. International Journal of General Medicine, 6, 189-200. https://doi.org/10.2147/IJGM.S42953

[7] Wakana, S., Caprihan, A., Panzenboeck, M.M., Fallon, J.H., Perry, M., Gollub, R.L., Hua, K, Zhang, J., Jiang, H., Dubey, P., Blitz, A., van Zijl, P. and Mori, S. (2007) Reproducibility of Quantitative Tractography Methods Applied to Cerebral White Matter. NeuroImage, 36, 630-644. https://doi.org/10.1016/j.neuroimage.2007.02.049

[8] Kroenke, K., Spitzer, R.L. and Williams, J.B. (2001) The PHQ-9: Validity of a Brief Depression Severity Measure. Journal of General Internal Medicine, 16, 606-613. 
https://doi.org/10.1046/j.1525-1497.2001.016009606.x

[9] McIntosh, A.M., Maniega, S.M., Lymer, G.K.S., McKirdy, J., Hall, J., Sussmann, J.E., Bastin, M.E., Clayden, J.D., Johnstone, E.C. and Lawrie, S.M. (2008) White Matter Tractography in Bipolar Disorder and Schizophrenia. Biological Psychiatry, 64, 1088-1092. https://doi.org/10.1016/j.biopsych.2008.07.026

[10] Gigante, A.D., Bond, D.J., Lafer, B., Lam, R.W., Young, L.T. and Yatham, L.N. (2012) Brain Glutamate Levels Measured by Magnetic Resonance Spectroscopy in Patients with Bipolar Disorder: A Meta-Analysis. Bipolar Disorder, 14, 478-487. https://doi.org/10.1111/j.1399-5618.2012.01033.x

[11] Alexander, S.P., Benson, H.E., Faccenda, E., Pawson, A.J., Sharman, J.L., Speddingn, M., Peters, J.A. and Harmar, A.J. (2013) The Concise Guide to PHARMACOLOGY 2013/14: Ligand-Gated Ion Channels. British Journal of Pharmacology, 170, 1582-1606. https://doi.org/10.1111/bph.12446

[12] Nakato, Y., Abekawa, T., Ito, K., Inoue, T. and Koyama, T. (2011) Lamotrigine Blocks Apoptosis Induced by Repeated Administration of High-Dose Methamphetamine in the Medial Prefrontal Cortex of Rats. Neuroscience Letters, 490, 161-164. https://doi.org/10.1016/j.neulet.2010.11.028

[13] Mark, A. (2006) Are Cross-Species Measures of Sensorimotor Gating Useful for the Discovery of Procognitive Cotreatments for Schizophrenia? Dialogues in Clinical Neuroscience, 8, 9-16.

[14] Takahashi, H., Hashimoto, R., Iwases, W., Ishii, R., Kamio, Y. and Takeda, M. (2011) Prepulse Inhibition of Startle Response: Recent Advances in Human Studies of Psychiatric Disease. Clinical Psychopharmacology and Neuroscience, 9, 102-110. https://doi.org/10.9758/cpn.2011.9.3.102

[15] Inoue, T., Inagaki, Y. and Kitazumi, K. (2012) Mechanism of Action of the Mood Stabilizer Lamotrigine. Japanese Journal of Clinical Psychopharmacology, 15, 735-747.

[16] Cooke, S.F. and Bliss, T.V. (2006) Plasticity in the Human Central Nervous System. Brain, 129, 1659-1673. https://doi.org/10.1093/brain/awl082

[17] Pomeroy, S.J., Ndikumana, R. and Cavanagh, J.P. (2017) Lamotrigine Induced Lymphadenopathy: Case Report and Literature Review. International Journal of Pediatric Otorhinolaryngology, 98, 82-84. https://doi.org/10.1016/j.ijporl.2017.04.047 